\title{
Erratum to: Bulk motion-independent analyses of water diffusion changes in the brain during the cardiac cycle
}

\author{
Tomoya Nakamura $\cdot$ Tosiaki Miyati $\cdot$ Harumasa Kasai $\cdot$ Naoki Ohno \\ Masato Yamada $\cdot$ Mitsuhito Mase $\cdot$ Masaki Hara $\cdot$ Yuta Shibamoto $\cdot$ \\ Yuriko Suzuki $\cdot$ Katsuhiro Ichikawa
}

Published online: 24 February 2010

(C) Japanese Society of Radiological Technology and Japan Society of Medical Physics 2010

Erratum to: Radiol Phys Technol (2009) 2:133-137

DOI 10.1007/s12194-009-0056-3

Equation (2) appeared incorrectly in the article cited above.

The correct equation is as follows:

$\mathrm{FA}=\sqrt{\frac{3}{2}} \frac{\sqrt{\left(\lambda_{1}-D\right)^{2}+\left(\lambda_{2}-D\right)^{2}+\left(\lambda_{3}-D\right)^{2}}}{\sqrt{\lambda_{1}^{2}+\lambda_{2}^{2}+\lambda_{3}^{2}}}$

The online version of the original article can be found under doi:10.1007/s12194-009-0056-3.

T. Nakamura $(\bowtie)$

Department of Radiology,

Tokai University Hachioji Hospital,

1838 Ishikawa, Hachioji 192-0032, Japan

e-mail: nakamura.tomoya@hachioji-hosp.tokai.ac.jp

T. Miyati $\cdot$ N. Ohno $\cdot$ K. Ichikawa

Division of Health Sciences,

Graduate School of Medical Science, Kanazawa University,

5-11-80 Kodatsuno, Kanazawa 920-0942, Japan

H. Kasai · M. Yamada · M. Hara · Y. Shibamoto

Department of Radiology,

Nagoya City University Hospital,

1 Kawasumi, Mizuho, Mizuho-ku, Nagoya 467-8602, Japan

M. Mase

Department of Neurosurgery and Restorative Neuroscience,

Nagoya City University Graduate School of Medical Sciences,

1 Kawasumi, Mizuho, Mizuho-ku,

Nagoya 467-8601, Japan

Y. Suzuki

MR Clinical Science, Philips Medical Systems,

2-13-37 Kounan, Minato-ku, Tokyo 108-8507, Japan 